\title{
Collaborative software solutions and data standards for ptychographic Imaging
}

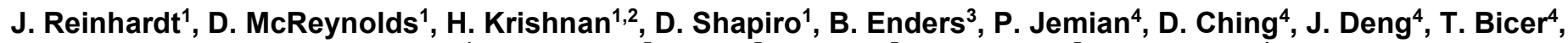 \\ N. Schwarz ${ }^{4}$, A. Barbour ${ }^{5}$, W. Hu ${ }^{5}$, D. Allan ${ }^{5}$, D. Gavrilov ${ }^{5}$, A. Hexemer \\ ${ }^{1}$ Advanced Light Source, Lawrence Berkeley National Laboratory, Berkeley, CA 94720, United States ${ }^{2}$ Center for Advanced \\ Mathematics for Energy Research Applications (CAMERA), Lawrence Berkeley National Laboratory, Berkeley, CA 94720, United \\ States ${ }^{3}$ National Energy Research Scientific Computing Center (NERSC) Lawrence Berkeley National Laboratory, Berkeley, CA \\ 94720, United States ${ }^{4}$ Advanced Photon Source, Argonne National Laboratory, Lemont, IL 60439, United States, ${ }^{5}$ National \\ Synchrotron Light Source II, Brookhaven National Laboratory, Upton, NY 11973, United States
}

$$
\text { jreinhardt@lbl.gov }
$$

Recent developments in 4th generation light sources and high-speed detectors are leading to rapid growth in data rates and data volumes, increasing the demand for automated data collection, handling/reduction/storage, and analysis processes. In combination with limited in-person access to experimental setups in times of the pandemic, portable and user-friendly tools for remote access as well as improved workflows are critical for enabling scientists from various disciplines to leverage ptychographic imaging to answer scientific questions.

With the growing popularity of ptychography, a broad range of data formats, acquisition schemes, and algorithms has been developed over the years, e.g. [1-3]. Whereas this variety has been advantageous to tackle different real-world deviations from the ideal ptychographic model such as partial incoherence [4], positioning errors [5], broad-bandwidth radiation [6], or multi-scattering [7], it also complicates the comparability and reproducibility of results. With ptychography being established as an everyday workhorse technique at many instruments around the world, it is important to find common ground and establish standards to support reliable algorithm and collaborative software development addressing the big data challenges of today and the future.

In this presentation, I will cover recent cross-facility efforts [8] to develop and promote data standards for ptychography. Furthermore, I will give an overview of ongoing software development at the Advanced Light Source in collaboration with the other DOE light sources for building data acquisition and analysis tools leveraging existing python packages with an outlook for future progress in terms of remote access and workflows.

[1] Enders B., \& Thibault P., (2016). Proc Math Phys Eng Sci. 472(2196) 20160640.

[2] Wakonig K., Stadler H.-C., Odstrčil M., Tsai E. H. R., Diaz A.,Holler M.,Usov I., Raabe J., Menzel A., \& Guizar-Sicairos M. （2020). Journal of Applied Crystallography, 53(2) 574-586

[3] Favre-Nicolin V., Girard G., Leake S., Carnis J., Chushkin Y., Kieffer J, Paleo P. \& Richard M.-I. (2020). J. Appl. Cryst. 53, 1404-1413

[4] Thibault P. \& Menzel A. (2013). Nature 494, pages 68-71

[5] Maiden A.M., Humphry M.J., Sarahan M.C., Kraus B. \& Rodenburg J.M., (2012). Ultramicroscopy, 120, 64-72

[6] Enders B., Dierolf M., Cloetens P., Stockmar M., Pfeiffer F. \& Thibault P., (2014). Appl. Phys. Lett. 104, 171104

[7] M Kahnt, Grote L, Brückner B., Seyrich M., Wittwer F., Koziej D. \& Schroer C. G., (2021). Sci Rep 11, 1500

[8] Data Solution Task Force Pilot https://www.bnl.gov/newsroom/news.php?a=216902

\section{Keywords: ptychography; microscopy; scientific software development; data formats; synchrotron;}

This work was supported by the U.S. Department of Energy, Office of Science, Office of Basic Energy Science, under the Data Solution Task Force Pilot.

The work was partially funded through the Center for Advanced Mathematics for Energy Research Applications (CAMERA), which is jointly funded by the Advanced Scientific Computing Research (ASCR) and Basic Energy Sciences (BES) within the Department of Energy's Office of Science, under Contract No. DE-AC02-05CH11231.

This research used resources of the National Energy Research Scientific Computing Center (NERSC), a U.S. Department of Energy Office of Science User Facility located at Lawrence Berkeley National Laboratory, operated under Contract No. DE-AC02$05 \mathrm{CH} 11231$.

This work was supported by the Advanced Photon Source, a US Department of Energy (DOE) Office of Science User Facility operated for the DOE Office of Science by ANL under contract No. DE-AC02-06CH11357.

This research used resources of the National Synchrotron Light Source II, a U.S. Department of Energy (DOE) Office of Science User Facility operated for the DOE Office of Science by Brookhaven National Laboratory under Contract No. DE-SC0012704. 